\title{
Recomendações Atuais para Vigilância Ativa no Câncer de Próstata
}

\author{
Current Recommendations on Active Surveillance for Prostate \\ Cancer
}

André Bacellar Costa Lima ${ }^{*}$, Ana Cristina Silva Bomfim ${ }^{1}$, Camila Severiano Vasques $^{1}$, Sergiane Amaral Gadelha ${ }^{1}$, Thereza Inês Barbosa Soares Flores ${ }^{1}$, Thiago Kaique Nunes Monteiro ${ }^{1}$, Thiago Santos Vieira ${ }^{1}$, Tércia Vilasboas Reis ${ }^{1}$, Érico Strapasson ${ }^{1}$

${ }^{1}$ Departamento de Oncologia Clínica do Hospital Santa Izabel - Santa Casa da Bahia; Salvador, Bahia, Brasil

Correspondence addresses: Dr. André Bacellar Costa Lima andre.bacellar@medicos. oncoclinicas.com

Received: September 21, 2021

Revised: October 29, 2021

Accepted: November 15, 2021

Published: December 28, 2021

Data Availability Statement: All relevant data are within the paper and its Supporting Information files.

Funding: This work was the result of author's initiative. There was no support of research or publication funds.

Competing interests: The author has declared that no competing interests exist.

Copyright

C 2021 by Santa Casa de Misericórdia da Bahia.

All rights reserved.

ISSN: 2526-5563

e-ISSN: 2764-2089

DOI: 10.35753
O câncer de próstata é uma das malignidades mais comuns ao redor do mundo. $O$ rastreamento e o diagnóstico precoce seguem sendo pilares para cura dos pacientes de risco desfavorável. No entanto, há uma preocupação a respeito do tratamento excessivo dos tumores de menor risco, onerando o sistema de saúde e causando potenciais efeitos colaterais irreversíveis em homens cuja qualidade de vida e sobrevida não seriam afetados pela doença. A vigilância ativa (VA), estratégia iniciada nas últimas duas décadas, é o tratamento de escolha nos pacientes com câncer de próstata de risco muito baixo,baixo e em alguns selecionados com risco intermediário favorável. Os principais protocolos de VA contemporâneos variam entre si nas suas coortes, manejos no monitoramento e no melhor momento para a intervenção. No entanto, os reportes de mortalidade câncer-específica e taxas de doença metastática são extremamente baixos no seguimento mediano de médio prazo (5-10 anos). Estes desfechos parecem estar intimamente associados aos diferentes critérios de seleção, monitoramento e intervenção, sugerindo que a estratégia da VA pode ser individualizada baseando-se no nível de risco aceitável para os pacientes, levando em consideração suas preferências pessoais. Novas tecnologias, como a ressonância nuclear magnética multiparamétrica e testes moleculares parecem tornar a VA mais segura. A utilização da VA, embora em crescimento, permanece altamente variável nos diferentes países e a educação continuada para segurança desta estratégia segue um desafio para que seu uso seja cada vez mais difundido.

Palavras-chave: Câncer de Próstata; Vigilância Ativa; Diagnóstico; Tratamento; Ressonância Nuclear Magnética Multiparamétrica.

Prostate cancer is one of the most common malignancies around the world. Screening and early diagnosis continue to be pillars for the cure of unfavorable risk patients. However, concerns exist regarding overtreatment of lower-risk tumors, burdening the health system and causing potentially irreversible side effects in men whose quality of life and survival would not be affected by the disease otherwise. Active surveillance (AS), a strategy initiated in the last two decades, is the treatment of choice in patients with prostate cancer at very-low and low risks, and in some selected patients with favorable intermediate risk. The current AS protocols differ in their cohorts, monitoring management and triggering for intervention. However, reports of cancer-specific mortality and metastatic disease rates are extremely low in the intermediate-term 
(5-10 years). These outcomes seem to be closely associated with different selection, monitoring, and intervention criteria, suggesting that AS strategy can be individualized based on the acceptable level of risk for patients, taking into account their personal preferences. New technologies such as multiparametric nuclear magnetic resonance and molecular testing seem to make AS safer. The use of AS, although growing, remains highly variable in different countries. The continuing education for the safety of these protocols remains a challenge for its use to be increasingly widespread.Keywords: Prostate Cancer; Active Surveillance; Diagnosis; Treatment; Multiparametric Magnetic Resonance Imaging.

\section{Introdução}

O câncer de próstata é o segundo câncer mais comum em homens no mundo, atrás somente do câncer de pele não melanoma. Nos Estados Unidos, as estimativas para 2021 são cerca de 248.530 novos casos. ${ }^{1}$ No Brasil, estimaram-se 65.840 novos casos em 2020, correspondendo a $29,2 \%$ dos tumores incidentes no sexo masculino; com um número de mortes de 15.983 em $2019 .^{2}$ As mortes associadas ao câncer de próstata são quase exclusivamente relacionadas ao paciente com câncer de risco desfavorável. Apesar do rastreamento ter aumentado o número de pacientes de alto risco tratados, este também está inexoravelmente associado ao diagnóstico excessivo de tumores localizados que dificilmente prejudicariam a qualidade e expectativa de vida caso não fossem tratados inicialmente.

O câncer de próstata localizado pode ser classificado de acordo com o risco de recorrência ou metástase após o tratamento, e os tumores de risco muito baixo, baixo ou intermediário favorável quando bem selecionados podem ser candidatos à estratégia de vigilância ativa (VA). Esta estratégia assume que selecionando os pacientes com métodos acurados e monitorandoos adequadamente, pode-se detectar a progressão da doença subclínica em momento oportuno, de modo que o tratamento será ainda com intuito curativo e não será mais mórbido do que no tempo do diagnóstico. As morbidades relacionadas ao tratamento definitivo com cirurgia ou radioterapia, como incontinência urinária e disfunção erétil, além dos custos envolvidos, são alguns dos motivos em adotar a VA. Outra estratégia conservadora para manejo desses pacientes é a chamada espera vigilante ("watchful waiting'), em que o manejo dos sintomas na progressão é a prioridade, em detrimento à cura. A classificação de risco associada a fatores como idade, expectativa de vida, presença ou ausência de comorbidades e preferência do paciente ajudam a definir a conduta terapêutica a ser adotada, tornando a VA ou mesmo a espera vigilante ainda mais atraente nos pacientes mais idosos e com expectativa de vida menor que 10 anos. ${ }^{3,4}$

O estudo PIVOT, realizado em pacientes com câncer de próstata localizado, já demonstrava que não existia ganho de sobrevida global nos pacientes de baixo risco submetidos a prostectomia radical (PR) em comparação com a VA. ${ }^{5}$ A segurança da VA nessa população é corroborada por diversos estudos. De fato, dados mostram que homens com câncer de baixo risco (ISUP grau 1) acompanhados por longo período em VA têm menos de $1 \%$ de risco de metástases e mortalidade câncer-específica de $0,1 \%$ em 15 anos. ${ }^{6}$ A força desses dados fez com que essa estratégia mais conservadora fosse mais difundida na comunidade médica e incorporada na prática clínica, especialmente na última década. ${ }^{7}$ Nos Estados Unidos, em 2000-2001, somente 6,2\% dos homens com baixo risco eram seguidos com VA ou espera vigilante, o que aumentou um pouco em 2004-2006 para 10,2\%, chegando a 40,4\% em 2010-2013, em dados mais recentes. ${ }^{8}$

Este trabalho aborda as recomendações atuais da VA no câncer de próstata, enfatizando as ferramentas clínicas disponíveis atualmente para melhor seleção do paciente candidato, como a ressonância magnética e testes genéticos, assim como a variância existente nos programas e diretrizes de monitoramento e gatilhos para indicar o tratamento. Além disso, este trabalho trata das controvérsias relacionadas à inclusão na 
VA de alguns grupos especiais, como pacientes mais jovens ( $<60$ anos), afrodescendentes e com tumores de risco intermediário favorável.

\section{Seleção de Pacientes}

O câncer de próstata localizado é classificado quanto ao risco de metástase e recorrência em muito baixo, baixo, intermediário favorável, intermediário desfavorável, alto e muito alto risco (Tabela 1). ${ }^{3}$

Essa classificação de D'Amico, sugerida pelo National Comprehensive Cancer Network (NCCN) considera o escore de Gleason, valor do PSA e o tamanho do tumor, foi utilizada por alguns dos principais programas de VA (Tabela 2). ${ }^{9-12}$

Os critérios mais estritos foram os utilizados no Johns Hopkins, ${ }^{6}$ baseados nos critérios de Epstein para câncer clinicamente insignificante $(\mathrm{T} 1 \mathrm{c}$, densidade de PSA $<0,15 \mathrm{ng} / \mathrm{mL}$; gleason $\leq 6 ; \leq 2$ fragmentos positivos com envolvimento tumoral $\leq$ $50 \%$ ), enquanto critérios mais abrangentes foram utilizados pelo Royal Marsden ${ }^{12}$ e Universidade de Toronto 9 , em que admitiam Gleason 3+4.

A VA é a proposta terapêutica padrão para os pacientes de muito baixo e baixo risco, ${ }^{3}$ mas outros fatores podem ajudar a refinar a seleção do paciente para VA, como a imagem da ressonância nuclear magnética (RM) multiparamétrica da próstata e seu papel em guiar a biópsia, presença de variantes histológicas de alto risco, histórico familiar, perfil genômico, densidade do PSA e, sobretudo, a preferência do paciente. Pacientes com risco intermediário favorável também são candidatos a VA, porém nestes a análise dessas outras características assume ainda mais importância na decisão terapêutica.

A RM da próstata surgiu como um importante componente para otimizar a seleção de pacientes

Tabela 1. Classificação de risco do câncer de próstata localizado (NCCN).

\section{Grupo de risco}

Muito baixo

Baixo

Risco intermediário

Intermediário favorável

Intermediário desfavorável

Alto

Muito alto

\section{Critérios}

Estádio T1c e escore de Gleason $\leq 6$ (ISUP Grau 1) e PSA $<10 \mathrm{ng} /$ $\mathrm{mL}$ e menos de 3 fragmentos de biópsia positivos com $\leq 50 \%$ de comprometimento em cada fragmento e densidade do PSA $<0,15$ $\mathrm{ng} / \mathrm{mL} / \mathrm{g}$.

Estádio $\leq \mathrm{T} 2 \mathrm{a}$ e escore de Gleason $\leq 6$ (ISUP Grau 1) e PSA $<10$ $\mathrm{ng} / \mathrm{mL}$. Excluídos os pacientes de muito baixo risco.

Estádio T2b ou T2c, escore de Gleason $3+4=7$ (ISUP Grau 2) ou $4+3=7$ (ISUP Grau 3) ou PSA 10-20 ng/dL.

Apenas um critério de risco intermediário, e Gleason 6 (ISUP Grau 1) ou $3+4=7$ (ISUP Grau 2) e menos de $50 \%$ de biópsias comprometidas.

Dois ou três critérios de risco de intermediário, escore de Gleason 4 $+3=7$ (ISUP Grau 3) e/ou $\geq 50 \%$ de biópsias comprometidas.

Estádio T3a, ou escore de Gleason 8 (ISUP Grau 4), ou escore de Gleason 9 ou 10 (ISUP Grau 5), ou PSA $>20 \mathrm{ng} / \mathrm{mL}$.

Apresenta pelo menos um dos seguintes critérios: Estádio T3b ou T4 ou padrão do escore de Gleason primário 5 (ISUP Grau 5), 2 ou 3 critérios de alto risco, ou mais de 4 fragmentos com Gleason escore 8-10 (ISUP Grau 4 ou 5). 
Tabela 2. Critérios de seleção para vigilância ativa.

\begin{tabular}{lcccccc}
\hline Programa & Estádio & $\begin{array}{c}\text { PSA } \\
(\mathbf{n g} / \mathbf{m l})\end{array}$ & $\begin{array}{c}\text { Gleason } \\
\text { Fohns Hopkins }\end{array}$ & $\begin{array}{c}\text { Fragmentos } \\
\text { positivos }\end{array}$ & $\begin{array}{c}\text { \% de câncer } \\
\text { no fragmento }\end{array}$ & $\begin{array}{c}\text { Densidade } \\
\text { do PSA }\end{array}$ \\
\hline Universidade de Toronto $^{9}$ & - & $\leq 10$ & $\leq 6$ & - & $\leq 50$ & $<0,15$ \\
& $<\mathrm{T} 2 \mathrm{a}$ & $\leq 10$ & $\leq 6$ & $\leq 2$ & - & - \\
PRIAS $^{10}$ & $\leq \mathrm{T} 2$ & $\leq 10$ & $\leq 6$ & $\leq 2$ & - & - \\
UCSF$^{11}$ & $\leq \mathrm{T} 2$ & $\leq 10$ & $\leq 6$ & $\leq 33 \%$ & 50 & $<0,2$ \\
Royal Marsden $^{12}$ & $\leq \mathrm{T} 2$ & - & $\leq 6$ & $\leq 50 \%$ & - & - \\
& & $<15$ & $\leq 3+4$ & $\leq 50 \%$ & - & - \\
\hline
\end{tabular}

PRIAS, Prostate Cancer Research International Active Surveillance; UCSF, University of California San Francisco; *expectativa de vida $<10$ anos.

para VA. Seu papel é localizar o tumor para fins de estadiamento e estratificação de risco, maximizar a acurácia da biópsia em detectar tumor clinicamente significante e ainda minimizar possíveis efeitos adversos de repetidos procedimentos. Dados de prostatectomia em pacientes outrora candidatos a VA sugerem que a biópsia inicial subestima a classificação de risco do paciente em cerca de 20$30 \%$ e este índice pode cair para 10-20\% quando direcionada pela RM. ${ }^{13}$ A RM é capaz de detectar cânceres clinicamente significativos com volumes $\geq 0,5 \mathrm{~mm}$, usando imagens ponderadas em $\mathrm{T} 1 \mathrm{e} \mathrm{T} 2$, associadas com informações funcionais de contraste dinâmico e difusão (Figura 1). Câncer clinicamente significativo é definido na patologia/histologia como escore de Gleason $\geq 7$ (incluindo $3+4$ com componente proeminente, mas não predominante, de Gleason 4) e/ou volume $\geq 0,5 \mathrm{~mm}$ e/ou extensão extraprostática. O sistema de PI-RADS foi criado para padronizar a notificação dos exames de RM com a definição de câncer clinicamente significativo (PIRADS 4 e 5), correlacionando-se com a patologia para aplicações clínicas e de pesquisa. ${ }^{14}$ Embora a biópsia deva ser considerada para PIRADS 4 ou 5, mas não para PIRADS 1 ou 2, o PI-RADS não inclui recomendações para o manejo, pois estas devem levar em consideração outros fatores, incluindo histórico laboratorial e clínico, preferências e experiências locais e diretrizes do centro. Por exemplo, em estudo randomizado que comparou a biópsia guiada por RM versus guiada por ultrassonografia, a chance de detecção de tumor clinicamente significativo nos PIRADS 3, 4 e 5 foi de 12, 60 e $83 \%$, respectivamente. Neste estudo, $28 \%$ dos pacientes no grupo da RM tiveram resultados do exame que não sugeriam câncer e, portanto, foram poupados da biópsia. ${ }^{15}$

Outro fator importante na seleção dos pacientes para VA é a análise criteriosa da histologia. A presença de componente intraductal, que não é um componente do escore de Gleason, pode estar associado a mutações de genes de recombinação homóloga de reparo do DNA (ex. BRCA2, ATM). Estas mutações estão associadas a tumores mais agressivos e maior risco de reclassificação do tumor em pacientes submetidos a VA, e pacientes com histologia intraductal foram excluídos de alguns programas de VA. ${ }^{16} \mathrm{O}$ padrão cribriforme invasivo representa o Gleason 4 e é biomarcador conhecido de prognóstico ruim, e sua presença também excluiu pacientes de programas de VA em algumas diretrizes.

Homens com mutações herdadas em genes de recombinação homóloga de reparo do DNA como BRCA1/2, ATM e CHEK2 são mais propensos a abrigar câncer de próstata agressivo e devem ser alertados para este risco quando incluídos em protocolos de VA. ${ }^{16}$ Estes testes devem ser indicados 
Figura 1. Ressonância nuclear magnética multiparamétrica.

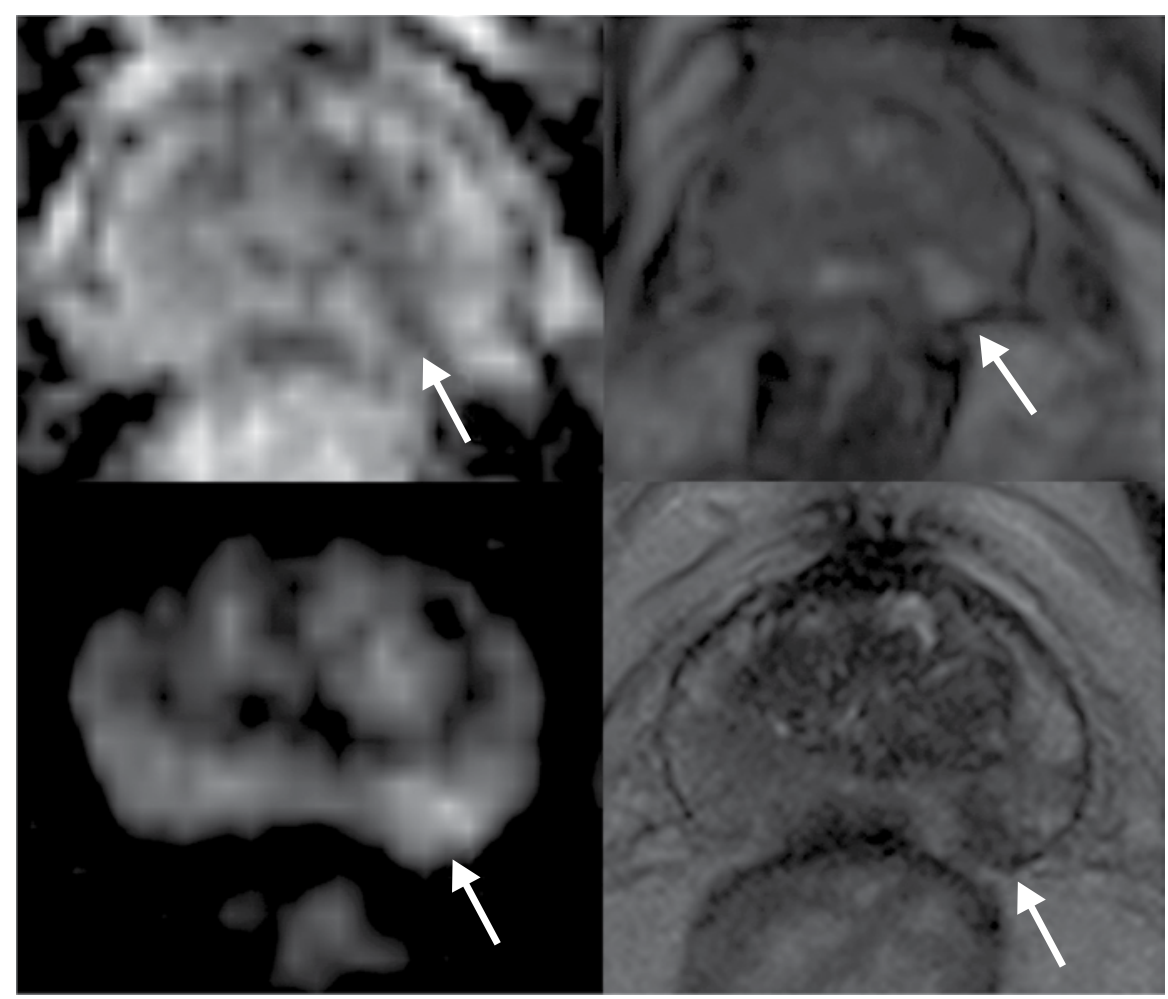

Zona periférica: sinal difusamente heterogêneo em T2. Nódulo com baixo sinal em T2, marcada restrição à difusão e realce precoce pós-contraste, situado na região posteromedial do terço médio do lobo esquerdo medindo $0,7 \mathrm{~cm}$, achado suspeito para neoplasia prostática clinicamente significante (PI-RADS 4).

nos pacientes com história familiar positiva para síndromes de predisposição ao câncer ou caso apresente histologia intraductal/cribriforme. Alterações germinativas estão presentes entre 4\% e $6 \%$ nos casos de câncer de próstata localizado e entre $12 \%$ e $17 \%$ dos pacientes com câncer de próstata avançado. Atualmente, não há consenso se pacientes com variantes patogênicas somáticas ou germinativas de genes de reparo do DNA devem ser excluídos de protocolos de VA, já que poucos estudos incluíram este perfil. No entanto, esta informação é importante no processo da decisão da conduta já que o risco de reclassificação do tumor é significativamente maior ao longo da vigilância. ${ }^{16}$

Os testes moleculares prognósticos que analisam o tecido prostático para biomarcadores específicos representam uma ferramenta útil na VA e são sugeridos pelo NCCN para os pacientes de risco baixo e intermediário favorável com expectativa de vida maior do que dez anos. ${ }^{3}$ Três testes são clinicamente disponíveis, embora ainda de difícil acesso no Brasil - Decipher, Prolaris e GPS. Enquanto os dois primeiros foram inicialmente desenvolvidos para predizer metástases após a prostatectomia radical, o OncotypeDx Genomic Prostate Score (GPS) foi desenvolvido para os pequenos fragmentos das biópsias por agulha considerando a natureza multifocal e heterogênea do tumor no intuito de ser útil no processo de decisão da VA. O Oncotype utiliza a técnica de RT-PCR para avaliar a expressão gênica de 17 genes de quatro diferentes vias, que são então combinados em um algoritmo para calcular o Genomic Prostate Score (GPS), com capacidade de predizer, independentemente, o risco de patologia desfavorável nos pacientes de risco baixo 
e intermediário. Como exemplo, um aumento de 5 unidades no GPS está associado a patologia adversa (HR 1,16, IC 95\% 1,06-1,26) e recidiva bioquímica (HR 1,10, IC 95\% 1,00-1,21) nos pacientes submetidos à prostatectomia postergada. ${ }^{17}$ Por outro lado, alguns estudos questionam se estes testes realmente adicionam valor aos critérios clínicos sozinhos, especialmente nos pacientes de baixo risco. ${ }^{18}$ Problemas relacionados a custo-efetividade, acesso, heterogeneidade tumoral, falta de estudos prospectivos randomizados para sua validação na VA, ainda limitam a utilização destes testes em nosso meio e estes devem ser limitados aos casos selecionados em que seu resultado poderia mudar a decisão terapêutica.

Outro biomarcador importante a ser considerado é a densidade do PSA (PSAD). Na coorte do programa de VA da University of California San Francisco (UCSF), uma PSAD de 0,10-0,15 foi associado à progressão da biópsia ( $v s<0,1$ : HR 2,06, IC 95\% 1,30-3,29), assim como foi uma PSAD $>0,15$ ( $v s<0,1$ : HR 2,83 , IC $95 \% 1,73-$ 4,62). ${ }^{11}$ Há ainda poucos dados a respeito do uso rotineiro de outros biomarcadores moleculares séricos e urinários, como o 4K score, o Prostate Health Index (PHI), o SelectMDx PCA3 e o MiProstate Cancer Score no contexto da VA e mais estudos são necessários. ${ }^{19}$
Homens manejados com VA tem a carga emocional de conviver com um câncer sem tratamento ativo. Essa ansiedade pode ser um fator significativo que contribui para a procura pelo tratamento e falha da VA. Na coorte da Universidade de Toronto, por exemplo, um terço dos pacientes procurou tratamento sem que a razão fosse a progressão da doença. ${ }^{9}$ Por outro lado, uma apropriada comunicação da equipe médica na educação do paciente a respeito do curso indolente da doença de bom prognóstico pode ajudar a evitar as complicações psicológicas relacionadas à VA e melhor adesão ao programa. ${ }^{20}$

\section{Monitoramento e Gatilhos para Intervenção}

O monitoramento sugerido pelo NCCN nos pacientes em VA compreende a realização de PSA e exame clínico a cada 6 meses, incluindo exame de toque retal anual; além de biopsia prostática e RM de próstata multiparamétrica no máximo a cada 12 meses, a menos que haja indicação clínica. ${ }^{3} \mathrm{~A}$ biópsia confirmatória entre 1-2 anos da vigilância é mandatória nesta diretriz e deve ser realizada dentro de 6 meses caso a biópsia inicial tenha menos de 10 fragmentos. Essas recomendações estão em linha com o que foi utilizado nos principais programas de VA (Tabela 3 ).

Tabela 3. Protocolos de monitoramento.

\begin{tabular}{|c|c|c|c|c|}
\hline $\begin{array}{l}\text { Programa: } \\
\text { intervalos de } \\
\text { monitoramento }\end{array}$ & $\begin{array}{c}\text { PSA } \\
\text { (meses) }\end{array}$ & $\begin{array}{c}\text { Exame clínico } \\
\text { (meses) }\end{array}$ & $\begin{array}{l}\text { Biópsia confirmatória } \\
\text { mandatória ( } \leq 1 \text { ano) }\end{array}$ & $\begin{array}{c}\text { Biópsias } \\
\text { subsequentes } \\
\text { (anos após) }\end{array}$ \\
\hline Johns Hopkins ${ }^{6}$ & 6 & 6 & Sim & 1 \\
\hline $\begin{array}{l}\text { Universidade de } \\
\text { Toronto }^{9}\end{array}$ & $\begin{array}{c}3 \\
\text { (por } 2 \text { anos) } \\
\text { após } 6\end{array}$ & - & Sim & $3-4$ \\
\hline PRIAS $^{10}$ & $3-6$ & - & Sim & 3 \\
\hline $\mathrm{UCSF}^{11}$ & 3 & 6 & Sim & $1-2$ \\
\hline Royal Marsden $^{12}$ & $\begin{array}{c}3-4 \\
\text { (por } 2 \text { anos) } \\
\text { após } 6\end{array}$ & $\begin{array}{c}3-4 \\
\text { (por } 2 \text { anos) } \\
\text { após } 6\end{array}$ & $\begin{array}{c}\text { Não } \\
(\leq 2 \text { anos) }\end{array}$ & 2 \\
\hline
\end{tabular}


É importante ressaltar o papel da biópsia confirmatória, obrigatória em todos os programas, devendo idealmente ser realizada dentro dos primeiros 18 meses do seguimento, uma vez que há chance de reclassificação em até $30 \%$ dos casos. ${ }^{13}$ Os protocolos mais atuais adicionaram a $\mathrm{RM}$ antecedendo a rebiópsia em um esforço para reduzir erros na coleta. Existem alguns dados que sugerem que uma RM negativa pode ser suficiente para renunciar à biópsia confirmatória,${ }^{21}$ entretanto, outros sugerem que a biópsia sistemática pode revelar câncer clinicamente significativo mesmo nestes casos. Como exemplo, no estudo randomizado ASIST que avaliou o uso da RM antes da biópsia confirmatória, $11 \%$ dos pacientes com RM negativa tiveram câncer clinicamente significativo detectado na biópsia sistemática; e a biópsia guiada pela RM perdeu $8 \%$ destes cânceres em toda a coorte. ${ }^{22}$ Portanto, nos protocolos atuais, a recomendação preponderante é da realização da biópsia confirmatória independentemente do resultado da RM.

A utilização da RM de maneira seriada durante a VA pode permitir intervalos aumentados entre biópsias, porém, é improvável que a RM permita omitir as biópsias subsequentes completamente, pois a sua capacidade de excluir com confiança a progressão do câncer ainda não está bem definida. ${ }^{22}$ Devido ao grande potencial demonstrado na detecção de cânceres de maior grau e potencial de guiar biópsias em lesões específicas, a maior parte dos protocolos incorporaram ao longo do tempo a RM no seguimento dos pacientes, porém ainda não foram descritos métodos uniformes para sua utilização. De fato, metanálise de sete estudos mostrou moderada acurácia da RM em detectar câncer ISUP $\geq 2$, com sensibilidade e especificidade de 69 e $78 \%$, respectivamente. Para uma probabilidade pré-teste de $20 \%$, o valor preditivo positivo foi de $44 \%$ e o valor preditivo negativo de $91 \%$, o que valoriza um teste negativo de RM para manter o paciente em VA..$^{23}$ Em outro exemplo, o escore do PIRADS inicial foi associado ao risco de reclassificação do tumor na coorte da UCFS. Homens com PIRADS 4 (versus PIRADS 1-2) têm risco 2,62 vezes maior (HR 2,62, IC 95\% 1,45-4,76) assim como PIRADS 5 (HR 4,38, IC 95\% 2,36-8,16). ${ }^{24}$

Enquanto o papel da RM ainda não está claramente definido, os protocolos contemporâneos ainda contam com biópsias subsequentes. A rebiópsia está indicada sempre que há sugestão de doença mais agressiva na RM, quando há alteração no exame clínico prostático ou alteração na cinética do PSA. Quando não ocorrem estas alterações, é prática clínica comum esperar por 2 anos até rebiópsia. Uma biópsia de seguimento negativa está associada a menor risco de reclassificação no futuro. ${ }^{25} \mathrm{Com}$ a associação da RM, uma abordagem aceitável no seguimento seria repetir a RM a cada 1-2 anos e a rebiópsia a cada 3-5 anos. Quando a expectativa de vida é menor do que dez anos ou o paciente encontrase em espera vigilante, a rebiópsia não está indicada.

A Tabela 4 detalha os gatilhos que indicam a intervenção curativa nos pacientes em VA utilizados nos principais programas de rastreamento. Caso a biópsia mostre doença ISUP $\geq 3$, ou caso o tumor seja encontrado em um número maior de fragmentos e uma porcentagem maior de determinado fragmento, a progressão da doença pode ter ocorrido e a intervenção dever ser considerada. O tempo de duplicação do PSA (PSADT) somente não parece ser um indicador confiável para detectar progressão de doença. $\mathrm{O}$ grupo de Toronto mostrou que a o PSADT $<3$ anos poderia substituir o critério de limite do PSA em 10 ou 20, por exemplo. Já o grupo do Johns Hopkins não encontrou associação entre o PSADT ou o PSAV e o risco de reclassificação do tumor.

\section{Desfechos}

Os cinco programas citados nesta revisão $0^{6,9-12}$ acompanharam mais de 6.000 pacientes com um follow-up mediano que variou entre 1,6 e 6,4 anos. Nos estudos com follow-up superior a $5^{6,9,11,12}$ anos observou-se uma necessidade de 
Tabela 4. Gatilhos para intervenção durante a vigilância ativa.

\begin{tabular}{|c|c|c|c|c|c|c|}
\hline Programa & Gleason & $\begin{array}{l}\text { Fragmentos } \\
\text { positivos }\end{array}$ & $\begin{array}{c}\text { Máxima \% } \\
\text { de câncer no } \\
\text { fragmento }\end{array}$ & PSAV & $\begin{array}{l}\text { PSADT } \\
\text { (anos) }\end{array}$ & $\begin{array}{c}\text { Pacientes } \\
\text { reclassificados } \\
(\%)\end{array}$ \\
\hline Johns Hopkins ${ }^{6}$ & $>6$ & $>2$ & $>50$ & - & - & $\begin{array}{c}36 \\
\text { Follow-up 5a }\end{array}$ \\
\hline $\begin{array}{c}\text { Universidade de } \\
\text { Toronto }^{9}\end{array}$ & Upgrade & - & - & - & $<3$ & $\begin{array}{c}23 \\
\text { Follow-up } 7 \mathrm{a}\end{array}$ \\
\hline PRIAS $^{10}$ & $>6$ & $>2$ & - & - & $<3$ & $\begin{array}{c}28 \\
\text { Follow-up 1.6a }\end{array}$ \\
\hline $\mathrm{UCSF}^{11}$ & $>6$ & $>33 \%$ & $>50$ & - & - & $\begin{array}{c}16 \\
\text { Follow-up 3.5a }\end{array}$ \\
\hline Royal Marsden $^{12}$ & $\geq 4+3$ & $>50 \%$ & - & $>1$ & - & $\begin{array}{c}22 \\
\text { Follow-up 5.7a }\end{array}$ \\
\hline
\end{tabular}

PSAV, velocidade do PSA (ng/mL/ano); PSADT, tempo de duplicação do PSA.

intervenção no quinto ano que variou entre 24$40 \%$. A mortalidade câncer-específica foi de 0 e $0,1 \%$ nas coortes da UCSF ${ }^{11}$ e da Johns Hopkins ${ }^{6}$, respectivamente - esta última com protocolos de inclusão e monitoramento mais rígidos. Mesmo nas coortes que permitiram inclusão do Gleason 3+4 e monitoramento mais flexível, como a Universidade de Toronto ${ }^{9}$ e o Royal Marsden ${ }^{12}$, a mortalidade câncer-específica foi baixa, com 1,5 e $0,4 \%$, respectivamente. Na coorte da Universidade de Toronto, ocorreu a maior incidência de metástases (2,8\%), sendo que $44 \%$ destes pacientes foi da população de Gleason 4. A taxa de recidiva bioquímica entre os pacientes que necessitaram de tratamento variou de 3-25\%.

\section{Grupos Especiais}

\section{$\underline{\text { Risco Intermediário }}$}

Apesar de controverso, a VA pode ser uma opção para pacientes estratificados como risco intermediário favorável, acima de 70 anos e/ ou com comorbidades limitantes, deixando sempre claro para o indivíduo a maior chance de apresentar progressão da doença. Embora não exista critérios claros para inclusão, estes pacientes devem ser bem selecionados, sendo que o Gleason predominante deve ser 3 , com $<50 \%$ de fragmentos da biópsia positivos e $\leq 1$ fator de risco intermediário (Tabela 1 ). ${ }^{3}$

A maior coorte de pacientes com risco intermediário foi descrita pela Universidade de Toronto, incluindo 132 pacientes com Gleason 7 (13\%), sendo $21 \%$ com Gleason 7 e/ou PSA $>10$ ng/mL. Observou-se que $44 \%$ dos pacientes com metástases vieram do grupo com Gleason 7 apesar de compreenderem apenas $13 \%$ da coorte. ${ }^{9}$

Na coorte do Royal Marsden, 7\% dos pacientes tinham Gleason 3+4 e, na análise multivariada, este foi associado a um risco 3,4 vezes maior de histologia adversa (Gleason $4+3$ ou $>50 \%$ de fragmentos positivos) na biópsia de seguimento, quando comparados aos Gleason 6. ${ }^{12}$ Por outro lado, dados da UCSF demonstraram uma diferença não significativa na taxa de progressão quando compararam pacientes de risco intermediário e baixo na sua instituição (61 versus $54 \%, \mathrm{p}=0,22)$. ${ }^{26}$

Acredita-se que, em breve, com o desenvolvimento de biomarcadores específicos e o refinamento dos exames complementares radiológicos, consiga-se selecionar melhor os pacientes de risco intermediário para a VA com maior segurança. 
Pacientes Jovens ( $\leq 60$ anos)

A inclusão de pacientes em VA implica em uma expectativa de vida $>10-15$ anos. No entanto, a literatura é limitada para desfechos $>$ 20 anos. A maior parte dos homens mais jovens diagnosticados são submetidos ao tratamento curativo imediato, porém a estratégia de VA pode também ser considerada nesta população. Não houve restrição de limite de idade na maior parte dos programas, porém, dados específicos relacionados à idade são pouco fornecidos. Em geral, os critérios de inclusão são pouco mais restritos nos pacientes jovens. Por exemplo, no Royal Marsden a inclusão de pacientes com Gleason 4 foi restrita aos $>65$ anos, enquanto os pacientes de baixo risco de até 50 anos foram incluídos. Não há dados que demonstram desfechos inferiores da VA na população jovem, além desta população sofrer uma maior redução na qualidade de vida após o tratamento comparada aos pacientes mais idosos. Adicionalmente, dados da coorte da UCSF sugere sua segurança: as taxas de sobrevida livre da reclassificação de Gleason nas biópsias de 3 e 5 anos foram de 73 e $55 \%$, respectivamente, nos homens $\leq 60$ anos, comparados a 64 e $48 \%$, respectivamente, nos homens $\geq 60$ anos $(\mathrm{p}<.01) .{ }^{27}$ No entanto, ainda há uma importante relutância na inclusão dos jovens na VA visto ser a literatura ainda limitada para desfechos $>20$ anos.

\section{$\underline{\text { Afrodescendentes }}$}

As coortes de VA atuais são limitadas pela ausência de diversidade de etnias. Afrodescendentes compreenderam apenas 7,4 e $13 \%$ nas coortes da Johns Hopkins e UCSF. Devido à conhecida agressividade do câncer de próstata nos afrodescendentes, existe uma preocupação acerca da sua inclusão em protocolos de VA. Entre 1.801 homens com câncer de muito baixo risco examinados na Johns Hopkins (14\% afrodescendentes), a etnia negra foi associada a um maior risco de patologia com características adversas (OR 3,23, p=0,03) e reclassificação patológica (OR 2,26, p=0,03) na análise multivariada. ${ }^{28}$ No entanto, os dados disponíveis ainda são insuficientes para recomendações contra a VA com os protocolos de seguimento atuais nesta população. No entanto, esta deve ser devidamente aconselhada quanto ao maior risco de progressão e desfechos desfavoráveis durante o seguimento.

\section{Podemos Desintensificar o Monitoramento na VA?}

A intensidade da vigilância pode ser ajustada com base na expectativa de vida do paciente e no risco de reclassificação. Pacientes com uma expectativa de vida inferior a dez anos (ou à medida que os homens submetidos a VA envelhecem ou desenvolvem comorbidades adicionais), a intensidade do monitoramento pode diminuir. Em alguns casos, a estratégia pode ser modificada para uma abordagem de espera vigilante, priorizando o tratamento de sintomas relacionados à progressão da doença, minimizando ou mesmo evitando as adversidades relacionadas ao tratamento.

Pacientes de baixo risco de progressão também são candidatos a uma vigilância menos onerosa. Por exemplo, na coorte da UCFS foram considerados fatores de risco para reclassificação do tumor durante a VA: PSAD $\geq 0,15$ (HR 3,37, IC 95\% 1,83-6,21), porcentagem de fragmentos positivos na biópsia (HR 1,27, IC 95\% 1,051,54 ) e alto escore genômico (HR 2,81, IC 95\% 1,21-6,52) na primeira biópsia da vigilância e, também, após 1-3 anos, após ajuste. PSAD $\geq$ 0,15 (HR 2,36, IC 95\% 1,56-3,56) e cinética do PSA (HR 2,19, IC 95\% 1,43-3,34) também foram associados com a reclassificação em 3-5 anos..$^{29}$ Outros fatores importantes são a idade (maior risco de progressão com $>60$ anos) e Gleason 3+4 nos estudos que permitiram a entrada destes pacientes. ${ }^{9,12}$ Sendo assim, estas informações podem ajudar a personalizar a VA apropriadamente nos pacientes. 


\section{Conclusão}

Após 20 anos de seguimento dos primeiros programas de VA, é possível quantificar os efeitos das variadas maneiras de seguir o paciente com segurança e esta estratégia vem difundindo-se cada vez na comunidade médica. Uma estratégia uniforme para seguimento do paciente seria muito atrativa, mas as ferramentas diagnósticas e prognósticas disponíveis atualmente ainda carecem de precisão para monitorar homens com diferentes riscos e preferências sob uma única ótica. A habilidade de individualizar o seguimento dos pacientes com seus riscos associados é imperativa. Os contínuos reportes dos programas de VA certamente irão ajudar a proporcionar o melhor cuidado nesta crescente população de pacientes.

\section{Referências}

1. American Cancer Society. Prostate cancer. Key statitiscs. Acessado em https://www.cancer.org/cancer/ prostate-cancer/about/key-statistics.html.

2. Instituto Nacional de Cancer. Estatística para Cancer de Próstata. Acessado em http://www.oncoguia.org.br/ conteudo/estatistica-para-cancer-de-prostata/5852/288

3. NCCNProstateGuideline; https://www.ncen.org/ professionals/physician_gls/pdf/prostate.pdf.

4. Sanda MG, Cadeddu JA, Kirkby E, Chen RC, Crispino $\mathrm{T}$, Fontanarosa J, et al. Clinically localized prostate cancer: AUA/ASTRO/SUO Guideline. Part I: Risk stratification, shared decision making, and care options. J Urol 2018:199:683.

5. Wilt TJ, Brawer MK, Jones KM, Barry MJ, Aronson WJ, Fox S, et al. Prostate cancer intervention versus Observation trial (PIVOT) study group. Radical prostatectomy versus observation for localized prostate cancer. N Engl J Med 2012:367;3.

6. Tosoian JJ, Mamawala M, Epstein JI, Landis, P, Wolf S, Trock BJ, et al. Intermediate and longer-term outcomes from a prospective active-surveillance program for favorable-risk prostate cancer. J Clin Oncol 2015;33(30):3379.

7. Dall'Era MA, Albertsen PC, Bangma C, et al. Active surveillance for prostate cancer: A systematic review of the literature. Eur Urol 2012;62:976-983.

8. Tosoian JJ, Carter HB, Lepor A, Loeb S. Active surveillance for prostate cancer: current evidence and contemporary state of practice. Nat Rev Urol 2016;13:205-215.
9. Klotz L, Vesprini D, Sethukavalan P, Jethava V, Zhang $\mathrm{L}$, Jain $\mathrm{S}$, et al. Long-term follow-up of a large active surveillance cohort of patients with prostate cancer. J Clin Oncol 2015;33:272-277.

10. Bul M, Zhu X, Valdagni R, Pickles T, Kakehi Y, Ranikko A, et al. Active surveillance for low-risk prostate cancer worldwide: the PRIAS study. Eur Urol 2013;63,597-603.

11. Welty CJ, Cowan JE, Nguyen H, Shinohara K, Perez $\mathrm{N}$, Greene KL, et al. Extended followup and risk factors for disease reclassification in a large active surveillance cohort for localized prostate cancer. J Urol 2015;193:807-811.

12. Selvadurai ED, Singhera M, Thomas K, Mohammed K, Woode-Amissah R, Horwich A, et al. Medium-term outcomes of active surveillance for localised prostate cancer. Eur Urol 2013;64:981-987.

13. Ploussard G, Salomon L, Xylinas E, Allory Y, Vordos D, Hoznek A, et al. Pathological findings and prostate specific antigen outcomes after radical prostatectomy in men eligible for active surveillance - does the risk of misclassification vary according to biopsy criteria? J Urol 2010;183(2):539.

14. Turkbey B, Rosenkrantz AB, Haider MA, Padhani AR, Villeirs G, Macura KJ, et al. Prostate imaging reporting and data system version 2.1: 2019 update of prostate imaging reporting and data system version 2. Eur Urol 2019;76(3):340.

15. Kasivisvanathan V, Rannikko AS, Borghi M, Panebianco V, Mynderse LA, Vaarala MH, et al. MRI-targeted or standard biopsy for prostate-cancer diagnosis. N Engl J Med 2018;378(19):1767.

16. Carter HB, Helfand B, Mamawala M, Wu Y, Landis P, $\mathrm{Yu} \mathrm{H}$, et al. Germline mutations in ATM and BRCA1/2 are associated with grade reclassification in men on active surveillance for prostate cancer. Eur Urol 2019 May;75(5):743-749.

17. Kornberg Z, Cooperberg MR, Cowan JE, Chan JM, Shinohara K, Simko JP, et al. A 17-gene genomic prostate score as a predictor of adverse pathology in men on active surveillance. J Urol 2019 Oct;202(4):702-709.

18. Lin DW, Zheng Y, McKenney JK, Brown MD, Lu $\mathrm{R}$, Crager $\mathrm{M}$, et al. 17-gene genomic prostate score test results in the Canary Prostate Active Surveillance Study (PASS) Cohort. J Clin Oncol 2020;38(14):1549.

19. Liu JL, Patel HD, Haney NM, Epstein JI, Partin AW, et al. Advances in the selection of patients with prostate cancer for active surveillance. Nat Rev Urol 2021;18:197-208.

20. Dall'Era MA, Konety BR, Cowan JE, et al. Active surveillance for the management of prostate cancer in a contemporary cohort. Cancer 2008;112(12):2664. 
21. Olsson H, Nordström T, Jäderling F, Egevad L, Vigneswaran HT, Annerstedt M, et al. Incorporating magnetic resonance imaging and biomarkers in active surveillance protocols - Results from the Prospective Stockholm3 Active Surveillance Trial (STHLM3AS). J Natl Cancer Inst 2021;113(5):632.

22. Klotz L, Loblaw A, Sugar L, Moussa M, Berman DM, Van der Kwast T, et al. Active surveillance magnetic resonance imaging study (ASIST): Results of a randomized multicenter prospective trial. Eur Urol 2019;75(2):300.

23. Guo R, Cai L, Fan Y, Jin J, Zhou L, Zhang K. Magnetic resonance imaging on disease reclassification among active surveillance candidates with low-risk prostate cancer: A diagnostic meta-analysis. Prostate Cancer Prostatic Dis 2015;18(3):221.

24. Chu CEA, Cowan JE, Lonergan PE, Washington SL, Fasulo V, de la Calle CM, et al. Diagnostic accuracy and prognostic value of serial prostate multiparametric magnetic resonance imaging in men on active surveillance for prostate cancer. Eur Urol Oncol 2021:Jan 19;S2588-9311(20)30203-0.
25. Rajwa P, Pradere B, Mori K, Ploussard G, Leapman MS, Shariat SF. Association of negative follow-up biopsy and reclassification during active surveillance of prostate cancer: A systematic review and metaanalysis. J Urol 2021;205(6):1559.

26. Cooperberg MR, Cowan JE, Hilton JF, et al. Outcomes of active surveillance for men with intermediate-risk prostate cancer. J Clin Oncol 2011;29:228-234.

27. Leapman MS, Cowan JE, Nguyen HG, Shinohara KK, Perez N, Cooperberg MR, et al. Active surveillance in younger men with prostate cancer. J Clin Oncol 2017:Jun 10;35(17):1898-1904.

28. Sundi D, Ross AE, Humphreys ED, Han M, Partin AW, Carter Schaeffer EM, et al. African american men with very low-risk prostate cancer exhibit adverse oncologic outcomes after radical prostatectomy: Should active surveillance still be an option for them? J Clin Oncol 2013:31,2991-2997.

29. Lonergan PE, Washington SL, Cowan JE, et al. Risk factors for biopsy reclassification over time in men on active surveillance for early stage prostate cancer. J Urol 2020 Dec;204(6):1216-1221. 